On the Movement of a Liquid Front in an Unsaturated, Fractured

Porous Medium, Part II. -.- Mathematical Theory

John J. Nitao

Earth Sciences Department

UCID- -21743

Lawrence Livermore National Laboratory

DE90 005414

Work pefformed under the auspices of the U.S. Department of Energy

by the Lawrence Livermore National Laboratory under contract

number W-7405-ENG-48. 


\title{
Un the Movement of a Liquid Front in an Unsaturated, Fractured Porous Medium, Part II. --- Mathematical Theory
}

\author{
John J. Nitao
}

\author{
Earth Sciences Department \\ Lawrence Livermore National Laboratory
}

\begin{abstract}
Work performed under the auspices of the U.S. Deparment of Encrgy by the Lawrence Livermore National Laboratory under contract number W-7405-ENG-48.
\end{abstract}

\begin{abstract}
A simplified equation of motion is derived for the flow of liquid through an idealized onc-dimensional fracture situated in an unsaturated imbibing porous medium. The equation is valid for the case where the matrix material has a much lower saturated conductivity than that of the fracture and the capillary tension is the matrix is sufficiently stronger than gravity. Asymptotic solutions and, in some cases, closedform solutions are given for the motion of the liquid front in a parallel fracture system. With the introduction of natural time constanes and dimensionless parameters, the flow behavior can be shown to possess various temporal flow regimes.

This work is part of the Nevada Nuclear Waste Storage (NNWS1) Project and is applicable to understanding some of the various physical parameters affecting liquid flow through a fracture in an unsalurated porous medium. and is particularly useful as a step in understanding the hydrological processes around a nuclear waste reposilory in an unsaturated environment as well as in other applications where unsaturated fracture flow conditions exist. The solutions are also selevant to numcrical model verification.
\end{abstract}




\section{Table of Contents}

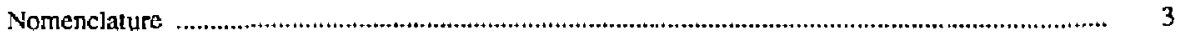

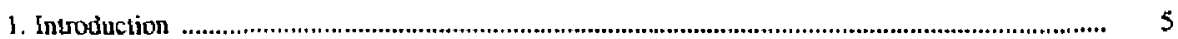

2. Derivation of the Goveming Equations .............................................................................. 5

3. Techniques for Analytic and Asymplotic Solution .............................................................. 8

4. imbibition Fluxes into the Matrix ....................................................................................... I2

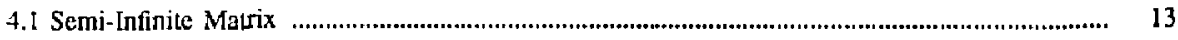

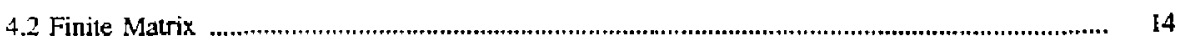

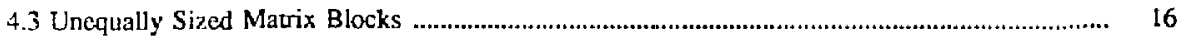

\$.4 Imbibition Kenel Function ............................................................................................... 18

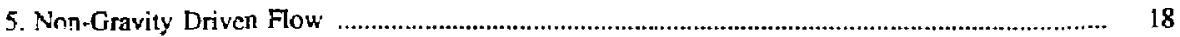

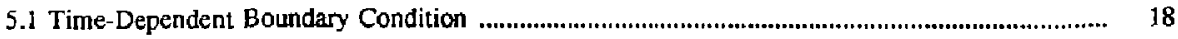

5.2 Constant Boundary Condition ................................................................................................. 19

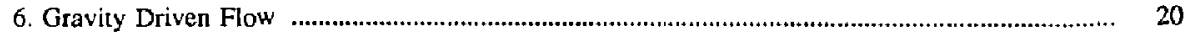

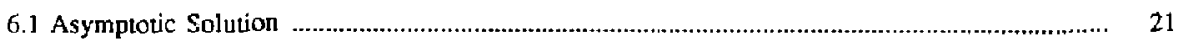

6.2 Comparison with Numerical Solutions to the Integro-Differential Equation .............................. 21

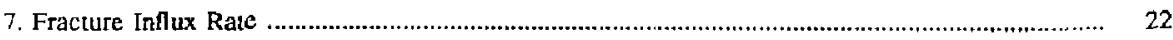

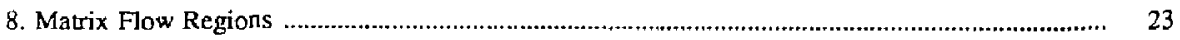

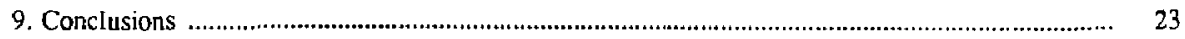

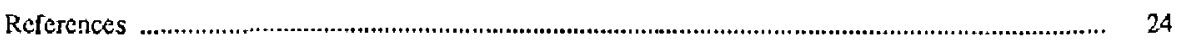

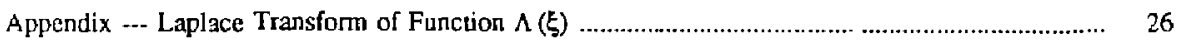




\section{Nomenclature}

Greek Symbols

$\beta$ cosine of the angle of inclination from the vertical

$\Gamma \quad$ the gamma function

$\lambda$ fracture storativity ratio, the initial unsaturated pore volume of the matrix relative to the volume of the fracture

$\Omega$ function $\Omega(y)$ denoting the time at which the fracture front first reaches point $y$

$\phi \quad$ matrix porosity

$\psi$ matrix capillary head

$\sigma$ matrix diffusivity, or cffecive matrix diffusivity

Roman Symbols

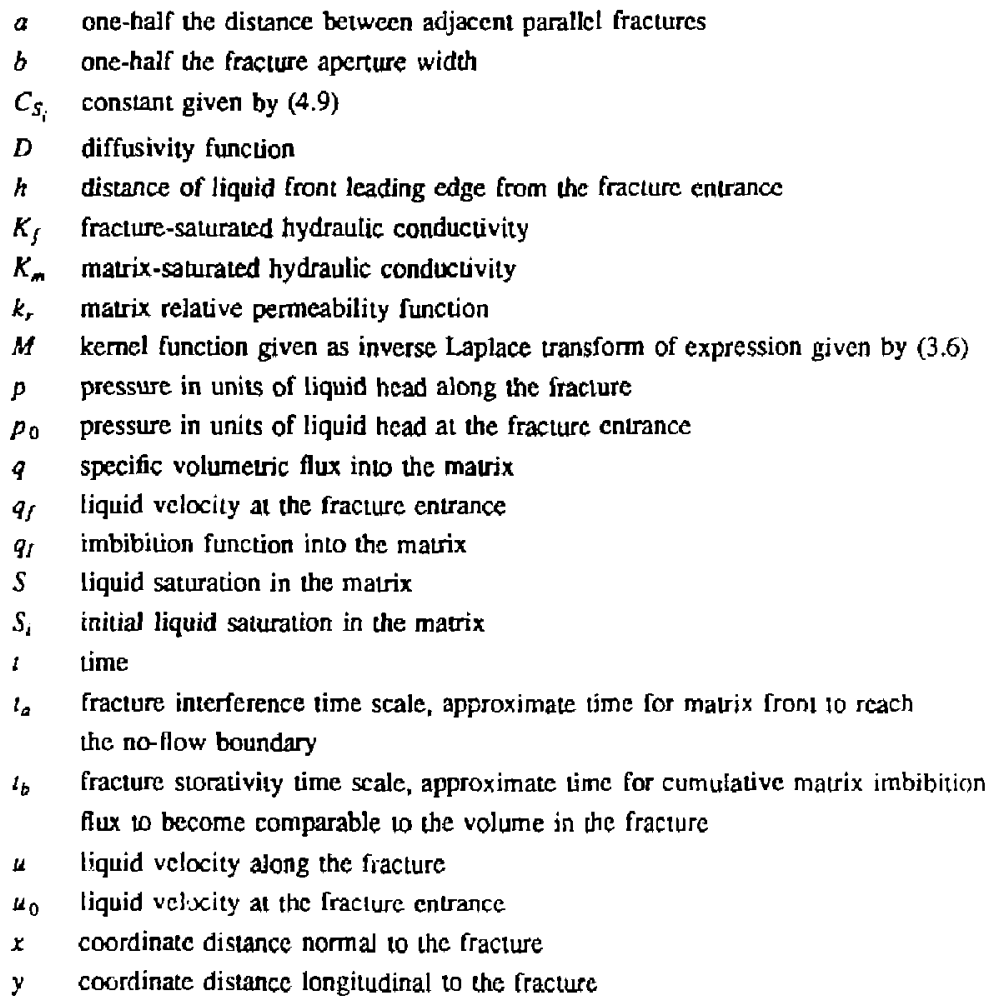


ya longitudinal distance along fracture from the entrance where flow region III begins (Figure 7, [Nitao and Buscheck, 1989])

$y_{6} \quad$ longiturinal distance along fracture from the enerance where flow region It begins

2 Laplace transform complex variable

2 flow region length 


\section{Introduction}

In this paper, we mathematically derive the conclusions that are described by Nitao and Buscheck [1989]. Under our simplifying assumptions, we show that the equations governing the flow down a one-dimensional fracture can be reduced to a single integro-differential equition in the fracture penetration. The asymptotic behavior of the solutions to this equation is shown to be directly related to the behavior of the Laplace transform of the matrix imbibition function. This function and its Laplace transform die derived for the case of a system of parallel fractures. For the case of constant boundary conditions, we demonstrate the existence of different flow regimes with the behavior of the solutions in each regime being described by its asymptotic expansion. In some cases a closed-form solution is derived. Some expressions for the solution are also given for the general problem with time-dependent boundary conditions.

\section{Derivation of the Governing Equations}

Using the simplifying physical assumplions given in Nitao and Buscheck [1989] we wil] derive the equations describing the movement of a liquid front in the fracture. We consider two separate types of boundary conditions at the entrance to the fracture: pressure head $p_{0}(l)$ and flux $u_{0}(l)$. It will be shown that, in each case, the governing equations reduce to a single equation for the location of the leading edge of the liquid front in the fracture with respect to the entrance to the fracture. This location will be referred to as the fracture penetration depth $h(t)$. These equations are integro-differential equations of the Volterra type [Burton, 1983].

In Nitao and Buscheck [1989] we saw that the following equations described the flow in the fracture and the matrix.

$$
\begin{aligned}
& \frac{\partial u}{\partial y}=-\frac{1}{b} a_{l}(t-\Omega(y)) \\
& u(y, t)=-K_{f}\left(\frac{\partial p}{\partial y}-\beta\right) \\
& \frac{d h(t)}{d t}=u(h(t), t) \\
& \Omega(h(t))=t
\end{aligned}
$$


We now reduce these equations to a single equation in the fracture penetration.

\section{Applied Flux Boundary Condition}

We first consider the problem where a given time-dependent flux $u_{0}(t)$ is applied to the opening of the fracture. The applicable boundary condition is

$$
u(0.1)=u_{0}(t)
$$

For this case, some care must be taken to see that the boundary condition is consistent with the assumptions of our derivation before applying the results for an applied flux boundary condition. Too great of a flux will create large pressure gradients in the fracure, thus violating the assumption of small gradients. Too small of a flux will result in a fracture front speed that is slower than the matrix fluxes which invalidates the assumption that the matrix streamlines are predominantly in a direction normal to the fracture.

We first imegrate (2.1) from $y=0$ to $y=h(t)$ and use (2.3) and (2.5). Making the change of variables $y=h(\xi)$ inside the integral, and using (2.4) we obtain

$$
\frac{d h(l)}{d t}=u_{0}(t)-\frac{1}{b} \int_{0}^{t} q_{l}(t-\xi) \frac{d h}{d \xi} d \xi
$$

which is the desired equation in $h(t)$.

\section{Applied Pressure Head Boundary Condition}

We now consider the case where the opening to the fracture at the ground surface is some known function of time $p_{1}(t)$ and the pressure at the leading edge of the fraciure front is kept at $p_{2}(t)$. The boundary condition is therefore

$$
p(y=0, t)=p_{1}(t) \quad p(y=h(t), t)=p_{2}(t)
$$

This set of conditions can, for example, be used to incorporate a constant capillary pressure drop at the leading edge of the front, or 10 include the effects of a constant head of water at the entrance. Note that since the form of the equations depends only on gradients in $p$, the solutions with these boundary conditions are equivalent to those satisfying

$$
\rho(y=0,1)=p_{0}(t) \quad p(y=h(t), t)=0
$$


where $p_{0}$ is defined as

$$
p_{0}(t)=p_{1}(t)-p_{2}(t)
$$

One must be careful that the magnitude of the pressure boundary condition $p_{0}$ is sufficienty small that the assumption of small pressure gradients in the fracture are satisfied.

We first solve (2.1) and (2.2) subject to (2.8). Substituting (2.2) into (2.1) we have

$$
\frac{\partial^{2} p}{\partial y^{2}}=\frac{1}{K, b} q(y, r)
$$

The solution to this equation that satisfies (2.8) can be shown to be

$$
p(y, t)=\left(1-\frac{y}{h}\right) p_{0}(t)+\frac{1}{b K_{f}}\left[F(y, t)-\frac{y}{h} F(h, t)\right]
$$

where we define

$$
F(y, t)=\int_{0}^{y} \int_{0}^{\eta} q(\mu, t) d \mu d \eta
$$

and where as before $h$ is the fraclure penetration depth. From Darcy's Law, (2,2), we have that the fluid velocity at the leading edge of the liquid fracture front is given by

$$
\begin{aligned}
\frac{d h}{d t} & =u(h, t) \\
& =K_{S}\left(\beta+\frac{p_{0}(l)}{h}\right)-\frac{1}{b}\left[\frac{\partial F}{\partial y}(h, t)-\frac{1}{h} F(h, t)\right]
\end{aligned}
$$

Using the change of variables of the form $\mu=h(\xi)$ in the same manner as wc have dene before, it can be shown that

$$
\frac{\partial F}{\partial y}(h, t)=\int_{0}^{t} q_{I}(t-\xi) \frac{d h(\xi)}{d \xi} d \xi
$$

Using a similar change of variables twice in the double integral $F(h, 1)$ and performing an interchange in the order of integration we obtain

$$
F(h, r)=h(t) \int_{0}^{1} q_{l}(t-\xi) \frac{d h(\xi)}{d \xi} d \xi-\int_{0}^{1} q(r-\xi) h(\xi) \frac{d h(\xi)}{d \xi} d \xi
$$

Substituting these expressions into (2.12) and using (2.3) we linally obtain

$$
h(t) \frac{d h(t)}{d t}=K_{f}\left(h(t) \beta+p_{0}(t)\right)-\frac{1}{b} \int_{0}^{t} q_{1}(t-\xi) h(\xi) \frac{d h(\xi)}{d \xi} d \xi
$$


which is the desired equation for $h(i)$. Note that this cquation is non-lincar in contrast to that for the specified flux boundary condition. The solution must also satisfy the initial condition

$$
h(0)=0
$$

since the penetration depth is taken to be zero at time zero. Note that in the special case when $p_{0}$ is identically zero, the trivial solution is one of the solutions to the problem, and a problem with nonuniqueness of the solutions may occur. It will be shown later that the non-trivial solution has the asymptotic expansion $h(t) \sim K_{f} f$, which can be used to start numerical solutions along the correct solution.

For the case of a horizontal fracture where gravity is not imponant we have

$$
h(r) \frac{d h(r)}{d t}=K_{f} p_{0}(t)-\frac{1}{b} \int_{0}^{1} q_{l}(t-\xi) h(\xi) \frac{d h(\xi)}{d \xi} d \xi
$$

We will later take advantage of the fact that this equation is linear in $h d h / d t$ and, as will be shown later, has a similar fonn to the equation (2.6) for the constant flux bounday vaiue problem.

The net specific volunietric flux $q_{f}(f)$ at the opening to the fracture, per unil area of opening. is an imporant quantity. By utilizing the same algebraic manipulations as usad above it can be shown to be given by

$$
\text { qf }(t)=\frac{d h}{d t}+\int_{0}^{1} \frac{1}{b} q_{1}(t-\xi) \frac{d h}{d \xi} d \xi
$$

\section{Techniques for Analytic and Asymptotic Solution}

We now describe some general methods for obtaining anat;ic and asymptotic solutions of the integro-differential equation derived in the previous section. The case where a specified flux boundary condition is applied at the entrance to the fracture and the case where a specified pressurc head exists, but with gravity neglected, can through renaming variables tout be reduced to the following form

$$
\frac{d g(l)}{d t}=f(s)-\frac{1}{b} \int_{0}^{t} q_{I}(t-\xi) \frac{d g(\xi)}{d \xi} d \xi
$$

where the definition of $g(l)$ and $f(l)$ depends on the boundary condition and is given by 
Case 1. (fiux boundary condition)

$$
g(t)=h(t) \quad f(t)=\mu_{0}(t)
$$

Case 2. (pressure head boundary condirion but no gravity, $\beta=0$ )

$$
g(t)=\frac{1}{2} h(t)^{2} \quad f(t)=K_{l} p_{0}(t)
$$

Equation (3.1) is linear and its solution $g(t)$ can be found by the taking the LaPlace transform. The Laplace cransform of $g(t)$, through the use of the convolution theoren [Docisch, 1974], can be shown to be

$$
\hat{g}(z)=\hat{M}(z) \hat{f}(z)
$$

Using the convolution thcoren again, we have

$$
g(t)=\int_{0}^{t} M(t-\xi) f(\xi) d \xi
$$

where, here, $M$ is the inverse Laplace transform of a Laplace transform function given by

$$
\hat{M}(z)=\frac{1}{z\left(1+\frac{1}{b} \hat{g}_{l}(z)\right)}
$$

with the 's denoting the Laplace transform operation. In the special case where the boundary condition is a constant in time, the function $f(t)$ will be a constant, say $f_{0}$, a:ld the solution reduces to

$$
f(t)=f_{0} \int_{0}^{r} M(\xi) d \xi
$$

Asymptotic forms for $g(1)$ can be most easily derived through looking at the asymplotic behavior of its corresponding Laplace transform. This was also the lechnique used by Philip [1968] in his study of infiltration into aggregated media. If the Laplace transform $\hat{g}(z)$ of a function $g(f)$ has the asymptotic expansion near $t=0$ of the form

$$
\xi(t) \sim e^{v} \sum_{k=0}^{n-1} a_{k} t^{k}+O\left(t^{n+v}\right) \quad t \rightarrow 0
$$

then its Laplace transform for large $z$ has the expansion

$$
\dot{\varepsilon}(z) \sim \sum_{k=0}^{n-1} a_{k} \frac{\Gamma(v+k+1)}{z^{k+v+1}} \quad z \rightarrow \infty
$$

and vice versa [Doetsch, 1974]. Thus, the behavior at sarly time can be deduced from the behavior of 
the Laplace trausform at infinity.

To delermire the solution behavior al late times, if the Laplace translorm $f(z)$ has the expansion about its extreme singularity $z^{*}$ of the form

$$
\hat{g}(z) \sim\left(z-z^{*}\right)^{-v} \sum_{k=0}^{n-1} A_{k}\left(z-z^{*}\right)^{k} \quad z \rightarrow z^{*}
$$

then $g(t)$ has the erpansion

$$
g(t)-t^{\nu-1} e^{i}+\sum_{k=0}^{n-1} \frac{B_{k}}{t^{k}} \quad t \rightarrow \infty
$$

where $B_{k}$ is defined as zero for $k$ such that $v-k$ is a negative integer but is otherwise given by

$$
B_{k}=\frac{A_{k}}{\Gamma(\nu-k)}
$$

The extreme singularity of a complex function is defined to be its singularity that has the largest real pait. Some functions can have more than one extreme singularity, in whish edrie there will be a sum uf expansions of the form (3.11) for each one. We refer the reader to Doetsch $\{19 ; 4)$ fcr more details.

Using (3.3) we can obtain the behavior of $\hat{g}(z)$ in terms of of $M(z)$ and $\hat{f}(z)$ and car, thercfore, derive the asymptotic expansions of $g(l)$ using the idacionships we have just described. The asymptotic form for small time is given by the form for $\hat{M}(z) \hat{f}(z)$. For large tume, one must find the extreme singularity of $g(z)$, that is, the singularity out of all those of either $\hat{M}$ or $\hat{f}$ that is the rightmost in the complex plane. The asymptotic behavior $o_{i}$ the product $\hat{M}(z) \hat{f}(z)$ must then be found at this point. Note that the peutt is not necessarily the extreme singularity of both factors, although it will be of one them, so that, in general, the time domain behavior of $M(t)$ and $f(t)$ at infinity can not be necessarily used to deduce the asymptotic behavior of $g(t)$. Analysis in the Laplace domain is essen:ial.

Since the Laplace transform function $\hat{M}$ depends on the transform of the imbibition function $\hat{q}_{1}$, the ask of the following section will be to derive this function and its asymptotic cxpansions. Since the extreme singularity of $\hat{M}$ will tum out to be at $z=0$, the behavior of $\hat{q}_{I}$ at this point will be important. Again, this behavior does not necessarily correspond to that of $q_{l}$ at $\rightarrow$ since the exireme singularity of its transform is not necessarily at the point of interest $z=0$. For exannple, an exponentially decreasing imbibition function has its extreme singularity on the negative real axis, not a: $2=0$. 
While the techniques given above apply to the problem with a pressure head boundary condition without gravity, the problem with gravity as given by (2.15) is non-linear and, therefore, can not be ats readily nor as thoroughly treated. Bul. asymptotic solutions can still be obtained by making trial subsitutions with various forms in $t$, equating like temns, and neglecting lower order terms as in tone in standard perturbation theory [Nayieh, 1973]. While this technique can give the learling terms of the expansion, in some cases, obtaining thr: higher order terms can cause problems. For example, in the rase of constant boundary condition the higher terms can be shown to be negative powers in I that go to infinity at $t=0$ and, hence, the integral in (2.15) diverges when these terms are substiluted. The way to avoid this problem is to perform the trial substitution in the Laplace transform domain instead of the time domain. By taking the Laplace transforin of (2.15), we can relate the transform of the function $h$ to the transform of the function squared as

$$
\hat{h^{2}}(z)=K_{f} \hat{M}(z)\left(\beta \hat{h}(z)+\hat{p}_{0}(z)\right)
$$

Let us restriet ourselves now to the case where $p_{0}$ is a constant so that $\dot{p}_{0}(z)=p_{0} / z$. The imbibition functions that we will encounter will be such that the resulting function $\hat{M}(z)$ will have an extreme singularity at $z=0$. Therefore, trial asymptotic forms for the solution $\hat{h}(z)$ that also have an extreme singularity at this point are a likely choice. These functions tum out to be those that in the time dor ain increase as positive powers in time for large time. Their Laplace transform behavior at $z=0$ is a power in $z$, and by expanding (3.12) in powers of $z$ and equating like terms, one can oblain an asymptotic expansion in $z$ and therefore in $t$. Since a mathematical proof as to the form of the expansion is not available, one must confirm the expansion ușing numcrical methods of solution. Again, the Laplace transform behavior of $\hat{q}_{l}$ at $z=0$ plays an important role in the analysis.

The asymptotic behavior of the specific flux $q_{f}$ into fraclure as given by $(2.17)$ can also be found using the Laplace transform. Its transform is given as

$$
\hat{q}_{f}(z)=\left[1: \frac{1}{b} \hat{q}_{J}(z)\right] z \hat{h}(z)
$$

Therefore, the asymptotic behavior can be found directly from the asymptotic behavior of the Laplace uransforms $\hat{q}_{l}(t)$ and $\hat{h}(t)$ near the extreme singularity $z=0$. 


\section{Imbibition Fluxes into the Matrix}

Equations (2.6) ard (2.15) both require knowledge of the imbibition lux $q_{5}$ from the fracture and into the matrix as a function of time. The behavior of is Laplace transform is important in that it determines the asymptotic behavior of the solutions. We will therefore derive in this section these imbibition functions under the assumptions stated in Nitao and Buscisck [1989] -..- the matrix streamlines are predominately in the direction normal to the fracture plane and the cffect of gravity is negligible in the matrix. These imbibition functions will first be öcrived for the case of a single fraclure with semi-infisite matrix blocks on both sides. Then, we consider the case of an infinite array of parallel and equally spaced fractures. The last case we consider is when the fractures are still parallel but not necessarily equally spaced from each other. Although the first two cases are included in the last case in the limil. their corresponding formulas will be useful in deriving simpler expressions.

Under our assumptions the flow in the matrix becomes onc-dimensional, and the equation for the saluration field $S$ reduces to

$$
\frac{\partial S}{\partial t}=\frac{\partial}{\partial x}\left[D(S) \frac{\partial S}{\partial x}\right]
$$

where $D$ is the diffusivity function given by $D(S)=\left(K_{\mathrm{m}} k_{\mathrm{r}} / \phi\right) d \psi / d S$. A saturated boundary candition occurs at a point on the fracture face for time $t$ from the time the liquid fracture front first arrives at that point. Here, we will take the time origin to be at zero. Therefore, the boundary condition at the fracture face $x=0$ of the matrix is

$$
s(x=0, r)=1 \quad t \geq 0
$$

Additional boundary conditions will be present depending on the problem. The initial saturation in the matrix is assumed to be uniform

$$
S(x, t=0)=S_{i} \quad x \geq 0
$$

The imbibition flux at $x=0$ is given by

$$
q_{l}(r)=-\phi D(1) \frac{\partial S}{\partial x}(x=0, t)
$$




\subsection{Semi-Infinite Matrix}

We now derive the one-dimensional imbibition flux into the end of a semi-infinile slab of matrix. The boundary conditions are

$$
S(x=0,1)=1 \quad S(x=\infty, 1)=S_{i}
$$

The Boltzmann transformation [Marshall and Holmes, 1979. p. 115]

$$
\eta=x / \sqrt{t}
$$

can be shown to reduce (4.1) to an equation with only $\eta$ as the deprindent variable; and, thercfore, the solution can be shown to be of the form

$$
S(x, l)=\left(1-S_{i}\right) F(x / \sqrt{t})
$$

where $F$ is a function that depends only on $S_{i}$ but not on $\phi$. Using (4.4) the imbibition flux is therefore equal to

$$
q_{i}(t)=\phi\left(1-S_{i}\right) C_{S_{i}} t^{-1 / 2}
$$

where we define the constant

$$
C_{S_{i}}=\frac{\partial F}{\partial \eta}(\eta=0)
$$

which, in general, depends on $S_{i}$. For constant diffusivity, $D(S)=\sigma$, it can be shown [Carslaw and Jaeger, 1959] that

$$
C_{S_{i}}=\sqrt{\frac{\sigma}{\pi}}
$$

By analogy, we define for non-constant $D(S)$, the "cffective diffusivity" $\sigma$ as

$$
\sigma=\pi C_{S_{i}}^{2}
$$

so that (4.10) holds for non-constant $D(S)$ the effective diffusivity is a function of the initial saturation $S_{i}$, unlike the constant $D(S)$ case. We have for the imbibition flux

$$
q_{l}(t)=\phi\left(1-S_{i}\right) \sqrt{\frac{\sigma}{\pi t}}
$$

A natural time constant that will arise is the time duration necessary, per unit lungitudinai area of fracture, for the imbibition front to invade a volume equal to the void volume of the fraclure. From $(4,12)$ this time is on the order of 


$$
i_{b}=\frac{\pi\left(b i\left(1-S_{i}\right) \phi\right)^{2}}{\sigma}
$$

The imbibition flux (4.8) can be rewritten in tenns of this constant as

$$
q_{1}(l)=\frac{b}{\sqrt{1 t_{b}}}
$$

The Laplace transform is given by

$$
\dot{q}_{1}(r)=b \sqrt{\frac{\pi}{2 t_{t}}}
$$

\subsection{Finite Matrix}

We now derive an imbibition flux function for the case of an infinite array of parallcl fractures having equal spacing $2 a$. The line at $x=c$ wil be a symmetry line and is assumed to be a no-flow boundary. The boundary conditions are then

$$
S(x=0, i)=J \quad \frac{\partial S}{\partial x}(x=a, i)=0
$$

The initial condition is, as before,

$$
S(x, l=0)=S_{i}
$$

In order to derive the saturation field we will have to assume, in contrast to the semi-infinite case, that the diffusivity $D(S)$ can be approximated as being equal to the effeclive diffusivity $\sigma$. This value will ensure that the imbibition fux will be accurate at least until the imbibition front reaches the no-flow boundary. After that the imbibition flux will decline and wil] not make a signifieant contribution to the total imbibition flux occuring along the entire fracture wall.

We first introduce the time constant

$$
\therefore=\frac{a^{2}}{\sigma} \pi
$$

which is the approximate time necessary for the imbibition front to reach the no-flow symmetry line between fractures. We will also use the time constant $t_{b}$ given by $(4,13)$.

The solution found by using the Fourier series melhod is [Kirkham and Powers, 1972; Carslaw and Jaeger, 1959] 


$$
S(x, i)=\left[1-2\left(1-S_{i}\right) \sum_{n=0}^{\infty} \frac{1}{c_{n}} e^{-c_{n}^{2} \pi t / t_{a}} \sin \frac{\varepsilon_{n} x}{a}\right]
$$

where $c_{n}=(2 n+1) \pi / 2$.

The imbibition fux into the malrix from the fracture is therciore

$$
q_{l}(t)=-\phi \sigma \frac{\partial S}{\partial x}(x=0, t)=\pi b \frac{\lambda}{r_{a}} \Lambda\left(\pi t / t_{a}\right) \quad t>0
$$

where we define the function $\Lambda$ by

$$
\Lambda(\xi)=2 \sum_{n=0}^{\infty} e^{-\left[\frac{2 n+1}{2} x\right]^{2} \xi}
$$

It can be shown (Appendix) that its Laplace transform $\hat{\Lambda}(z)$ is given by $\hat{\Lambda}(z)=\frac{1}{\sqrt{z}} \tanh \sqrt{z}$, and, hence,

$$
\frac{1}{b} \hat{q}_{l}(t)=\lambda \frac{\tanh \sqrt{l_{a} 2 / \pi}}{\sqrt{l_{a} 2 / \pi}}
$$

This function has two separate expansions

$$
\begin{aligned}
& \frac{1}{b} \hat{q}_{l}(z)-\lambda \sqrt{\frac{\pi}{2 t_{b}}}, \quad t_{a} z \gg 1 \\
& \frac{1}{b} \hat{q}_{l}(z)-\lambda\left(1-\frac{1}{3} \frac{t_{a} z}{\pi}\right), \quad t_{a} z \ll 1
\end{aligned}
$$

for large and small 2. They will be used later to deternine the fracture penctration at $t \ll t_{a}$ and $t>t_{a}$, respectively.

In order to gain a perspective on the function $\wedge$ from another direction, the solution to the saturation field by a different method, the methou of images, gives

$$
\zeta(x, t)=\sum_{n=0}^{\infty}(-1)^{n}[f(x+2 \pi a, l)+f((2 n+2) a-x, l)]
$$

where $f(x, t)$ is the solution [Carslaw and Jaeger, 1959] for the semi-infinite case given by

$$
S(x, r)=1-\frac{2\left(1-S_{i}\right)}{\sqrt{\pi}} \int_{0}^{x / 2 \pi \sigma t} e^{-\xi^{2}} d \xi
$$

The fesulting alternate expression for the imbibition flux is

$$
\frac{1}{b} q_{l}(l)=\frac{1}{\sqrt{t_{b}}}\left[1+2 \sum_{n=1}^{\infty}(-1)^{n} e^{-\frac{n^{2} t_{0}}{\pi t}}\right] \quad t>0
$$


Therefore,

$$
\frac{1}{b} q_{l}(l)-\frac{1}{\sqrt{\pi l_{b}}}+o\left[\frac{1}{\sqrt{\pi l / l_{a}}} e^{-\frac{1}{\left(\pi / l_{a}\right)}}\right] \quad t \ll l_{a}
$$

This expansion expresses the face that for small time the imbibition fux is approximately the same as for the semi-infinite matrix.

\subsection{Unequalły Sized Matrix Blocks}

We now consider the effect of non-equal spacing on the i;nlibition flux. In Figure 3 of Nitao and Buscheck [1989] we have a fracture that is part of an array of fmclures whose separations alternate between distances $2 a_{1}$ and $2 a_{2}$. The no-flow symmetry lines in the maurix are therefore $a_{1}$ from one side of the Iracture and $a_{2}$ from the other. Eact: side is allowed to have different material propertics $t_{1}$ and $\sigma_{k}(k=1,2)$, as well as initial saturation $S_{i k}$.

We introduce natural time constants analogous to those encountered for equidistant fracture systems.

$$
i_{b k}=\frac{\left[2 \sigma i\left(1-S_{i k}\right) \phi_{k}\right]^{2} \pi}{\sigma_{k}} \quad t_{a k}=\frac{a_{k}^{2}}{\sigma_{k}} \pi \quad k=1.2
$$

Note that there is a factor of two inside the brackets in the definition of $t_{b}$ which is not present in $l_{b}$. The $t_{b k}$ refer to each of the matrix blocks, singly, draining the entire fracture width $2 b$ by imbijition while $t_{b}$ refers to simultaneous imbibition into both matrix blocks. I.et us now assums that

$$
r_{01} \ll t_{02}
$$

If the matrix diffusivity $\sigma$ were equal on both sides of the fracture, this assumption would correspond physically to an array of fractures with separations alternating between a smalt distance apart and a large distance apart.

At any given point along the fracture the imbibition Rux $q_{l}(t)$ into the matrix, at lime $t$ from start of imbibition, can be writien as the sum of the hux into the two sides of the fracture. Using the expression $(4,2)$ for the imbibition flux into a finite matrix slab, the flux from the two sides of the fracture into a half-fracture is

$$
\frac{1}{b} q_{I}(t)=\pi\left[\frac{\lambda_{1}}{t_{a 1}} \Lambda\left(\pi t / t_{a 1}\right)+\frac{\lambda_{2}}{t_{a 2}} \Lambda\left(\pi t / t_{a}\right)\right]
$$


where the function $\Lambda(t)$ was defined in $(4.21)$, and where we define

$$
\lambda_{k}=\sqrt{\frac{t_{c k}}{t_{b k}}}
$$

We now determine some asymptotic expansions for $q_{l}$. For early times such that $p<<\ell_{a l}$, we have fro.T (4.28) that

$$
\frac{1}{b} q_{1}(t)-\frac{1}{\sqrt{t_{b 1}}}+\frac{1}{\sqrt{t_{b} 2}}=\left(t_{b}\right)^{-1 / 2}
$$

where $t_{b}$ is defined as the harmonic-roo! mean

$$
\frac{1}{\sqrt{t_{b}}}=\frac{1}{\sqrt{b_{b 1}}}+\frac{1}{\sqrt{t_{b 2}}}
$$

At intermediate lim,es, $t_{a 1}<t<t_{a 2}$, we have from $(4.20)$ and $(4.28)$,

$$
\frac{1}{b}=(t) \sim \pi \frac{\lambda_{1}}{l_{a 1}} \Lambda\left(\pi t / t_{a}\right)+\left(s t_{b 2}\right)^{-1 / 2}+O\left(\frac{1}{\sqrt{\pi l / t_{a 2}}} e^{-\frac{1}{\pi t / r_{d}}}\right)
$$

The contributior. of the imbibition flux into matrix block number 1 as given by the first term on the right decays exponentially wihh time while the second term, the fux into 2 , is dominant since it decays as a power in time.

Using (4.22) the Laplace transform is given by

$$
\frac{1}{b} \hat{q}_{f}(c)=\lambda_{1} \frac{\tanh \sqrt{t_{a 1} 2 / \pi}}{\sqrt{t_{a 1} / \pi}}+\lambda_{2} \frac{\tanh \sqrt{t_{a 2} 2 / \pi}}{\sqrt{t_{a 2} / \pi}}
$$

Each of the two terms is in the form of (4.22) so that they have expansions of the form (4.23) and (4.24). If we assume, without loss of generality, that $t_{a l} \leq t_{d 2}$, then we have the following expansions

$$
\begin{aligned}
& \frac{1}{b} \hat{a}_{1} \sim \sqrt{\frac{\pi}{a_{b} z}}, \quad z t_{a_{1}}>1 \\
& \frac{1}{b} \hat{q}_{I} \sim \lambda_{1}\left(1-\frac{1}{3} \frac{t_{a 1} z}{\pi}\right)+\sqrt{\frac{\pi}{t_{b 2} z}}, \quad z t_{a_{1}}<<1, \quad z t_{a_{1}}>1 \\
& \frac{1}{b} \hat{q}_{I} \sim \lambda-\frac{1}{3 \pi} z\left(\lambda_{1} t_{a l}+\lambda_{2} t_{a 2}\right), \quad z t_{a_{2}}<<1
\end{aligned}
$$

These will comespond to the threc low periods $t<<t_{a_{1}}, t_{a} \mid<<t<<t_{a 2}$, and $t_{a 2}<<t_{\text {, te }}$ pectively, of the fracture penctration solution $h(t)$. 


\subsection{Imbihition Kernel Function}

The imbibition kemel function function $M(t)$ given by $(3.6)$ has been summarized in Table I for the fracture geometries we have just considered.

Table I. Laplace Transform of the Imbibition Kernel Function

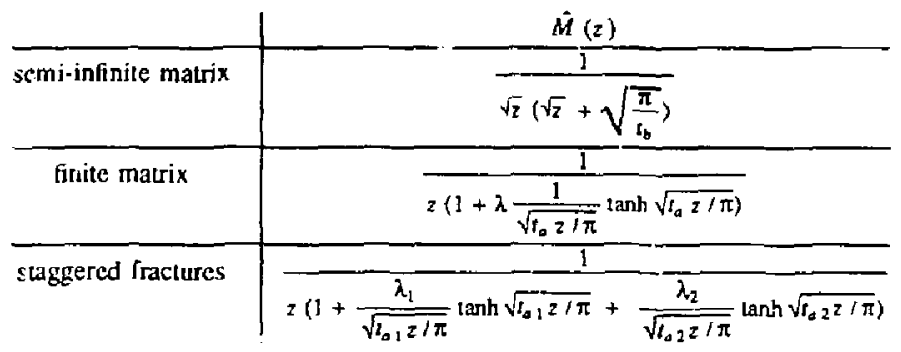

\section{Non-Gravity Driven Flow}

In this section we derive the solutions for the two types of boundary conditions, applied flux $u_{0}(t)$ and applied pressure head $p_{0}(t)$ with no gravity. Unter the appropriate transformation of varjables given by (3.2) and (3.3), the problems werc snown to reduic to the same equation (3.1). In this section we will arribe expressions for the solutions to this equation. Although the general timedependent bountary contition will be considered firsh, we will be particularly interested in the asymptotic behavior of the solutions in the case where the boundary condition is kept at a constant value.

\subsection{Time-Dependent Boundary Condition}

The Laplace transforms $\hat{M}(z)$ of the imbitition kemel function $M(l)$ were given is Table I for various geometrics. In the $\mathrm{ct}$ for a singie fracture with semi-infinite matrix the inverse Laplace transform is known and is given by [Abrahamowitz and Siegun, 1964, p. 1024]

$$
M(t)=e^{\pi / t_{b}} \operatorname{erfc} \sqrt{\pi t / t_{b}}
$$

where erjc is the complementary etror function. Therefore, (3.5) can be used to give an expressicn for the general solution to $g(i)$, and the penetration depth can be found by (3.2) and (3.3) for the 
approprials boundary condition.

For the case of a system of parallel fractures equally spaced, the kernel function can to found by taking the inverse uransform of the expression for $\hat{M}(z)$ given in Table I by the method of residues IDoctsch, 1974]. It is given as an infinite sum

$$
M(r)=\frac{1}{1+\lambda}+2 \lambda \sum_{n=1}^{\infty} \frac{1}{\zeta_{n}^{2}+\lambda+\lambda^{2}} e^{-\zeta_{n}^{2} \pi r / t_{a}}
$$

where the $\zeta_{n}$ are the roots of the equation

$$
\lambda \sin \zeta_{n}=\zeta_{n}, \quad n=1,2,3, \cdots
$$

in ascendin ${ }_{0}$ order. The $n$th term in the infinite sum corresponds to the exponentially decaying interference from the iracture that is the $n$th one away from the fracture of interest.

\subsection{Constanı Boundary Condition}

We now consider the solutions to (3.1) in the case where the boundary condition $f(t)$ is equal to a conerant $f_{0}$. Using (3.7), we find that the solution in the case of a single fracture with semi-infinitc matrix is the integral of (5.1). Using various integral equalities we have that

$$
g(l) / f_{0}=\frac{f_{b}}{\pi}\left[e^{\pi t i t_{b}} \operatorname{erf} c\left(\sqrt{\pi t / t_{b}}\right)-1+2\left(t / t_{b}\right)^{3 / 2}\right]
$$

For the case of a system of equidistant parallel fractures the solution is given by (3.7), which has as its Laplace transform $\dot{M}(z) / z$ where $A^{i}$ is taken from Table I. We use the method of resiudues to take the inverse transform of this expression to obtain

$$
g(i) / f_{0}=\frac{1}{1+\lambda} t+\frac{\lambda l_{a}}{3 \pi(1+\lambda)^{2}}-\frac{2 \lambda_{a}}{\pi} \sum_{n=1}^{\infty} \frac{1}{\zeta_{n}{ }^{2}\left(\zeta_{n}^{2}+\lambda+\lambda^{2}\right)} e^{-i_{n}^{2} \eta_{1} l_{1}}
$$

where the $\zeta_{n}$ are given by (5.3).

The asymptouic expansions of the solution can be most easily derived by looking at the asymptolic expansions of its Laplace transform. From the convolution theorem we have

$$
\dot{g}(z) / f_{0}=\frac{1}{z} \dot{M}(z)
$$

We consider the case of a system of fractures with staggered spacing. This case encompasses the other 
cases as limiting cases. From (4.37) to (4.39) the asymplotic expansions of $\hat{M}(z)$ and hence of $\hat{g}(z)$ can be found and are given in Table Il. The corresponding expansion for $g(f)$ is also shown.

Table IL. Expansions for $\frac{1}{z} \dot{M}(z)$ and $g(t) / f_{0}$

\begin{tabular}{|c|c|c|}
\hline Flow Period & $\frac{\hat{\xi}(z)}{f_{0}}=\frac{1}{z} \dot{M}(z)$ & $\frac{g(s)}{f_{0}}$ \\
\hline I. & $\begin{array}{l}z^{-2}-z^{-52 \sqrt{\pi / t_{b}}} \\
t_{b}^{-1} \ll<\end{array}$ & $\begin{array}{l}1-\frac{4}{3} \frac{1}{\sqrt{t_{b}}} t^{3 / 2} \\
t<t_{b}\end{array}$ \\
\hline II. & $\begin{array}{l}z^{-3 / 2} \sqrt{t_{b} / \pi}-z^{-1} t_{b} / \pi \\
z_{a} 1^{-1}<<z<s_{b}^{-1}\end{array}$ & $\begin{array}{l}\left.\frac{2}{\pi}\left\langle r_{b} t\right\}^{12}-t_{b}\right) \pi \\
t_{b}<<1<<t_{3}:\end{array}$ \\
\hline IIla. & $\begin{array}{l}z^{-3 / 2} \sqrt{d_{b 1} / \pi}-z^{-1}\left(1+\lambda_{1}\right) d_{b 2} / \pi \\
t_{a z^{-1}}<<z<<t_{a-1}^{-1}, z<<b_{b 2}{ }^{-1}\end{array}$ & $\begin{array}{l}\frac{2}{\pi}\left(t_{b 7} t\right)^{1 / 2}-\left(1+\lambda_{1}\right) t_{b 2} / \pi \\
t_{b} \ll r \ll t_{0} \cdot t_{b 2} \ll t\end{array}$ \\
\hline IIIt. & $\begin{array}{l}2^{-2} \frac{1}{1+\lambda}+z^{-1} \frac{\lambda_{1} t_{\Delta 1}+\lambda_{2} r_{3}}{3 \pi(1+\lambda)^{2}} \\
z<<t_{\Delta 2}{ }^{-1}\end{array}$ & $\frac{1}{1+\lambda} t+\frac{\lambda_{1} t_{01}+\lambda_{2} t_{a 2}}{3 \pi(1+\lambda)^{2}}$ \\
\hline IIla.! & $\begin{array}{l}z^{-2} \frac{1}{1+\lambda_{1}}+z^{-1} \frac{\lambda_{1} t_{01}}{3 \pi\left(1+\lambda_{1}\right)^{2}} \\
t_{a} z^{-1}<<2<<t_{a} 1^{-1}, z>>i_{b 2}{ }^{-1}\end{array}$ & $\begin{array}{l}\frac{1}{1+\lambda_{1}}+\frac{\lambda_{1} t_{a 1}}{3 \pi\left(1+\lambda_{1}\right)^{2}} \\
t_{b} \ll t \ll f_{a}, I_{b 1} \gg t\end{array}$ \\
\hline IIla. 2 & $\begin{array}{l}\text { same as for IIL.a } \\
t_{0} 2^{-1}<<z<<t_{b 2}{ }^{-1}\end{array}$ & $\begin{array}{l}\text { same as for IIl.a } \\
t_{b 2}<<t<<t_{a z}\end{array}$ \\
\hline
\end{tabular}

\section{c. Gravity Driven Flow}

The case where there is gravily driven flow with a pressure head byundary condlion possesses the governing equation given by (2.15). We will consider the solution when the boundary head is equal to some positive constant $p_{0}$. 


\subsection{Asymptotic Solution}

The asymplotic expansions to (2.15) were given in Table II of Nitao and Buscheck [1989|. They were obtained by substituting the expansiors for $\hat{M}(z)$ as given ill Table II of this paper into a version of $(3.12)$ given by

$$
\hat{h^{2}}(z)=K_{f} \hat{M}(z)\left(B \hat{l}(z)+\frac{p_{0}}{z}\right)
$$

We then try the following functionil form

$$
\hat{h}(z)-\alpha z^{-v}
$$

where $v>0$. This assumption is equivalent to assuming that the form of the solution is

$$
h(t) \sim \alpha:^{v-1} / \Gamma(v)
$$

By equating the leading terms in $z$ one can find the value of $\alpha$ and $v$. The higher-order term can be added and its coefficiemt and power can be found. This process is suaightorward if the twundary head $p_{0}$ is zero with respect to ambient Otherwise, one expects that the effects of the hoindary head will dorninate at early times when the head of ijquid in the ĩaciure is small with respect to the boundary head. And, at later times, when the converse becomes true the effects of the gravity head will become Jominant over the boundary head. Hence, the leading tem in the expansion for (6.1) will depend on the relative ragnitude of the head term $p_{0}(t)$ in $(2.15)$ to the liquid column head term $\beta h(t)$. The time al which the boundary head dominates can be estimated by comparing the expansion for $\beta h$ based on zero boundary head with the term $p_{\mathrm{p}}$. This determination was performed for cach of the flow periods I through III as shown in Table II of Nitao and Buscheck [1989].

\subsection{Comparison with Numerical Solutions to the Integro-Differential Equation}

In order to confirm the asymptotic solutions for the case of a constant boundary condition with: gravity, we have also found solutions to (2.15) numerically. Originally. we discretired the equation by the obvious procedure: the time rerivatives were replaced by first-order differences and the integral with a sum. However, we found that the errors due to the differences inside the sum can accumulate, requiring very small time steps to be taken to maintain accuracy. A belter method is to reduce the cquation 10 a system of two equations while laking $h$ and $d h / d t$ as separate dependent variables. In the end, 
this method was refined by using $h$ and $v$ as the dependent variables where $v$ is defined as

$$
v=\frac{1}{2} \frac{d h^{2}}{d l}-K_{f} \beta h
$$

Equation (2.15) with $p_{0}$ zero is then equivalent to

$$
v(1)=-\frac{1}{b} \int_{0}^{t} q_{1}(t-\xi]\left[v(\xi)+K_{f} \beta h(\xi)\right] d \xi
$$

This choice of variables eliminates the round-off error duc to subtraction of the $K_{f} \beta \mathrm{h}$ and the integral terms. These equations were discrelized in time as follows, where the superscripl refers to the time level,

$$
\begin{aligned}
\frac{1}{2}\left(h^{(n+1)}\right)^{2} & =\frac{1}{2}\left(h^{(n)}\right)^{2}+\Delta v^{(n+3)}\left(K_{f} \beta \dot{h}^{(n)}+v^{(n)}\right) \\
v^{(n+1)}= & -\frac{1}{b} \sum_{k=1}^{n} q_{1}\left(t^{(n+1)}-r^{(k)}\right)\left[v^{(k)}+K_{f} \beta h^{(k)}\right] \Delta s^{(k)} \\
& -\frac{1}{b} a_{i}\left(\frac{1}{2}\left(r^{(n+1)}-f^{(n)}\right)\right) \frac{1}{2}\left[v^{(n)}+v^{(n+1)}+K_{j}\left[1\left(h^{(n)}+h^{(n+1)}\right)\right] \Delta s^{(n+1)}\right.
\end{aligned}
$$

where

$$
\Delta t^{(k)}=t^{(k)}-t^{(k+1)}
$$

In Nitao and Buscheck [1989] comparisons were also made with solutions using a twodimensional unsaturated flow simulator. These simulations will be discussed in morc detail in a future repor.

\section{Fracture Influx Rate}

The expression for the Laplace transtorm of the specific fracture influx rate $q_{f}$ was given by (3.13). It requires the behavior of the Laplace transform for the imbibition function $q_{i}$ and the fracture penetration $h(t)$ which have been found in the previous sections. Thereforc, the asymptotic bchavior of qf can be found, as shown in tab]e $V$ of Nitao and Buscheck [1989], using the techniques in scction 3 that relate the asymptotic behavior of the Laplace transform to that of the ciginal function in the lime domain. 


\section{Matrix Flow Regions}

We briefly explain how the lengths of the various matrix Aow regions shown in Table VI of Nitao and Buscheck [1989] were derived. We first find the distances $y_{a}$ and $y_{b}$ (referring to Figurc 7 of Nitao and Buscheck [1989]) of the flow regions from the fracture entrance as funclions of ime. To find $y_{a}(t)$ we note that the time elapsed from when the fracture front first hits a point $y^{*}$ is given as $\left(-\Omega\left(y^{*}\right)\right.$. When this time increment equals $t_{a}$, the saturation front corresponding to this point has felt the no-flow boundary due to the neighboring fracture, according to the definition of $t_{a}$. Hence, the leading edge $y_{a}$ of the "saturated" flow region is at this point $y^{*}$. This may be expressed as

$$
t-\Omega\left(y_{a}(t)\right)=t_{a}
$$

Since $\Omega$ is the inverse function of the fracture penetration $h$, we have

$$
y_{a}(t)=h\left(t-t_{a}\right) .
$$

Similarly, $y_{b}$ is given as $y_{b}(t)=h\left(t-b_{b}\right)$. Hence,

$$
\begin{aligned}
& Z_{1}=h(t)-y_{b}=h(t)-h\left(t-t_{b}\right) \\
& Z_{2}=y_{b}-y_{a}=h\left(l-t_{b}\right)-h\left(t-t_{a}\right)
\end{aligned}
$$

By substituting the asymptotic expansions for $h(t)$ and dropping any higher-order terms one can obain expansions for $Z_{1}$ and $Z_{2}$.

In paricular, the entries in Table VI of Nitao and Buscheck [1989] were derived using the expansions for $h$ that are valid for $t>l_{a}$. The requirement that the arguments of $h$ in $(8 . l)$ and $(8.2)$ satisfy this condition on $t$ translates to having $t>2 t_{a}$.

\section{Conclusions}

We have found that the unsaturated flow of a liquid front in a fracture can, under certain situations, be described by a single integro-differential equation whose solution gives the location of the from in the fracture. This equation can be most satisfactorily treated by using Laplace transform techniqucs. Various asymptotic approximations can be derived which are sulficient to characterize the 
physical processes of the system including various flow periods, regions in the matrix saluration ficld, and the liquid flux into the fracture. Closed-form solutions were derived for some typess of boundary conditions.

The use of the Laplace transform has been found to be a very convenient deviec fo. the derivation of asymptotic solutions to our problem. The method is genesal enough to be applicable to other lypes of iv: hihicion functions in addition to those treated in this report.

In the special case of a single fracture with scmi-infinite matrix the analysis is applicable even when the matrix diffusivity is a non-constant function of saturation. In our more general analyses when the matrix is fittite, the nlatrix diftusivity has to be approximated by a constant effective diffusivity which is defined in terms of the expression for the imbibition flux in a semi-infinite system. Comparisons with numerical simulations indicate that this approximation gives good resuls for the test cases considered. The reason for the agreement stems from the fact that the frontal movement in the fracture depends on the matrix imbibition flux along the fract.ire wall and not on the actual form of the saturation field. Either the imbibition flux along the fracture is (1) nearly the same as for a semi-infinite matrix because the matrix front has not yet felt the no-flow boundary with the neighboring fracture, (2) is very small hecause the the matrix block is almost satusated, or (3) has values iniermediate to those in (1) and (2). In most cases, only a small amount of the net imbibiuon flux will be due to (2) and (3) which exnlains the applicability of the constant effectivc diffusivity approximation.

\section{References}

Abraharrowitz, M., and Stegun, 1.A., Handbook of Mashematical Functions, National Bureau of Standards, U.S. Govt. Prining Office, p. 2024 (1954).

NNA. 891120.0023

Buron, T.A., Volterra Integral and Differential Equations, Academic Press (1983). NNA.891109.044

Carslaw, H.S., and Jaeger, J.C., Conduction of Heat in Solids, second edicion, Clarendon Press, Oxford (1959). HQZ.870131.2201

Doalsch, G., Introduction to the Theory and Application of the Laplace Transform Springer-Vorlag. Fo. 238, 254-255 (1974). NNA.891120.0024 
Gradshieyn, I.S. and Rhyzik, I.M., Table of Iniegrals, Series, and Products, Academic Press, p. 37 (1980). NNA.891120.0025

Kirkham, D. and Powers, W.1., Advanced Soil Physics, Kriegcr Pub. Co. (1972). NNA.891109.0045

Marshall, T.J. and Holmes, J.W., Soil Physics, Cambridge Universily Press, p. 115 (1979). NNA.891128,0598

Nayfeh, A.H., Perturbation Methods, John Wiley, pp. 6-7 (1973). NNA.891120.0026

Nitao, J.J., and Buscheck, T.A., On the Movement of a Liquid Front in an Unsaturated, Fractured Porous Mediun, Part I., Lawrence Livermore Laboratory UCID Report (1989). NNA. 891130.0050

Philip, J.R. The Theory of Absorption in Aggrcgatcd Media, Auslralian Journal of Soil Research, vol. 6,1-19 (1968). NNA.891128.0581 
Appendix ... Laplace Transform of the Function $\wedge(\xi)$

It is well known that the Laplace transform of the exponential function $e^{-\alpha r}$ is given by Jif $(z+\alpha)$. Thus,

$$
\hat{\Lambda}(2)=2 \sum_{n=0}^{\infty} \frac{1}{z+((2 n+1) \pi / 2)^{2}}
$$

Integrating this function with respect to $\mathrm{z}$ stanting from $z=0$ gives

$$
\begin{aligned}
\int_{0}^{2} \hat{\Lambda}(\eta) d \eta & =2 \sum_{n=0}^{\infty} \ln \left[1+\frac{z}{((2 n+1) \pi / 2)^{2}}\right] \\
& =2 \ln \prod_{n=0}^{\infty}\left[1+\frac{z}{((2 n+1) \pi / 2)^{2}}\right]
\end{aligned}
$$

Using the idenlity [Gradstehyn and Ryzhik, p. 37]

$$
\cosh \zeta=\prod_{n=0}^{\infty}\left[1+\frac{\zeta^{2}}{((2 n+1) \pi / 2)^{2}}\right]
$$

we have

$$
\int_{0}^{x} \hat{\Lambda}(\eta) d \eta=2 \ln \cosh \sqrt{2}
$$

Taking the derivative,

$$
\hat{\Lambda}(z)=\frac{1}{\sqrt{2}} \tanh \sqrt{z}
$$


The following number is for Office of Civilian Radioactive Waste Management Records Management purposes only and should not be used when ordering this document:

Accession Number: NNA.891130.0051. 